\title{
Performance Analysis of Rectangular Patch Antenna with Dielectric Constants
}

\author{
Karuna Kumari. K , P. V. Sridevi
}

\begin{abstract}
The developments in communication systems, patch antennas play a very significant role in today's world of communication systems. The most commonly used micro strip patch antennas are Rectangular patch antennas.

The rectangular micro strip patch antenna parameters are analyzed for S-band frequency which is used for wireless communications $(2.0-2.5 \mathrm{GHz})$. In this design, performance parameters like V.S.W.R, Returnloss, are simulated and radiation patterns are observed.

Rectangular patch antenna is designed with uniform and non-uniform linear arrays. The uniform and non-uniform arrays are designed with Dolph-Tschebycheff.
\end{abstract}

Keywords : Rectangular micro strip patch antenna, uniform array and Dolph-Tschebycheff .

\section{INTRODUCTION}

Radiating element of rectangular patch antenna on one side of a dielectric substrate which has a ground plane on the other side of the antenna are shown in Fig.1.

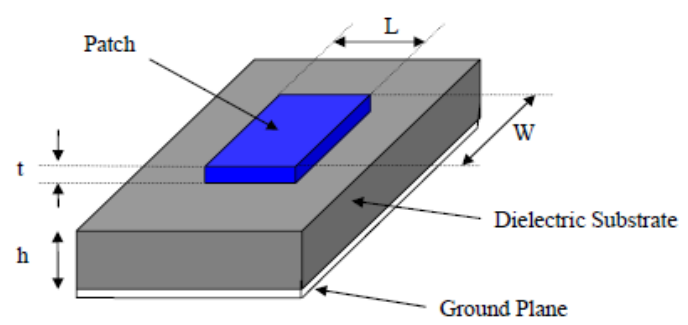

Fig.1. Rectangular antenna

The patch is made of conducting material such as copper or gold and can be found in various shapes. The radiating element $\&$ feed lines are fabricated using a process of photo etching which uses a photo resist and etchants to corrosively machine away selected areas on the dielectric substrate. Structure and side view of the rectangular microstrip patch antenna as shown in Fig. 2.

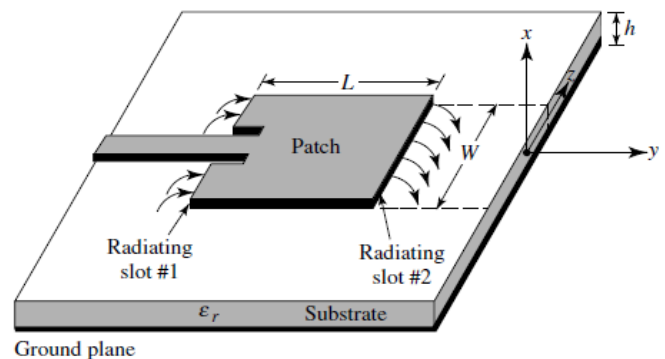

(a)Structure of Microstrip antenna

Revised Manuscript Received on December 13, 2019.

* Correspondence Author

Karuna Kumari *, Research Scholars, Dept. of ECE, AU College of Engineering, Andhra University, Vizag, A.P

Prof.P. V. Sridevi,, Dept. of ECE, AU College of Engineering, Andhra University, Vizag, A.P

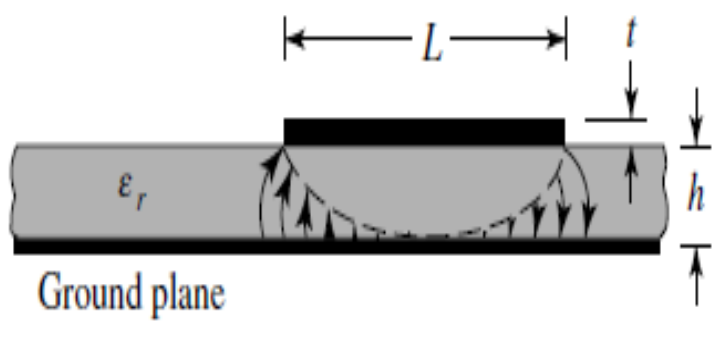

(b) side view of Rectangular antenna

Fig. 2. structure and side view of antenna Some of the other common shapes in micro strip antennas $n$ shown in Fig.3.
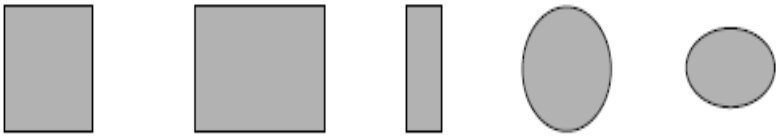

(a) Square

(b) Rectangular

(c) Dipole

(d) Circular

(e) Elliptical
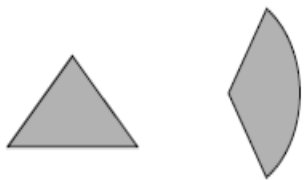

(f) Triangular

(g) Disc sector
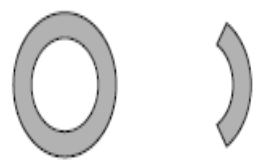

(h) Circular ring

(i) Ring sector
Fig. 3.Diff. shapes of patch

\section{RECTANGULAR MICROSTRIP PATCH GEOMETRY}

Rectangular microstrip antenna consists of a very thin such that $t<\lambda_{0}$ (where $\lambda_{0}$ is the free - space wavelength and $\mathrm{t}$ is the patch thickness) metallic strip is placed a small fraction of a wavelength, dielectric height $h$,is usually $0.003 \lambda_{0} \leq h \leq$ $0.05 \lambda_{0}$ above a ground plane. The $\mathrm{L}$ is the length of the patch is usually $0.3333 \lambda_{0}<L<0.5 \lambda_{0}$

\section{RECTANGULAR PATCH DESIGN EQUATIONS}

A. The width of the microstrip patch antenna

$$
W=\frac{c}{2 f_{r} \sqrt{\frac{\varepsilon_{r}+1}{2}}}
$$

$c=$ The speed of light

$W=$ Patch width

$\varepsilon_{r}=$ Substrate dielectric constant

$f_{r}=$ Resonant frequency

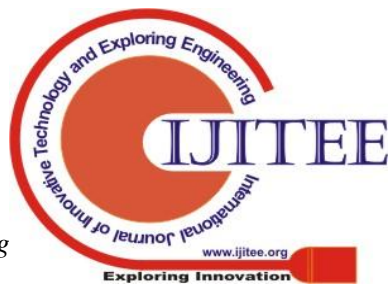


B. Effective dielectric constant $\varepsilon_{\text {reff }}$

$$
\varepsilon_{r s f}=\frac{\left(\varepsilon_{r}+1\right)}{2}+\frac{\left(\varepsilon_{r}-1\right)}{2}+\left[1+12 \frac{h}{W}\right]^{1 / 2}
$$

C. The effective length $L_{e f f}$

$$
L_{\text {eff }}=L+2 \Delta L
$$

Rectangular patch length

$$
L_{e f f}=L+2 \Delta L
$$

D. The resonance frequency:

$$
f_{r}=\frac{C}{2 \sqrt{\varepsilon_{r s f f}}}\left[\left(\frac{m}{L}\right)^{2}+\left(\frac{n}{W}\right)^{2}\right]^{\frac{1}{2}}
$$

E. The radiated power:

$$
p_{\text {rad }}=\frac{\left|V_{0}\right|^{2}}{2 \pi \eta_{0}} \int_{0}^{\pi}\left[\frac{\sin \left[\frac{k_{0} W}{2} \cos \theta\right]}{\cos \theta}\right]^{2} \sin ^{3} \theta d \theta
$$

Rectangular patch antenna, the $x-y$ plane $\left(\theta=90^{\circ}, 0 \circ \leq \varphi \leq\right.$ $90^{\circ}$ and $\left.270^{\circ} \leq \varphi \leq 360^{\circ}\right)$ is the principal $E$-plane. The radiated fields

$$
E_{\Phi}{ }^{t}=\frac{+j k_{0} W V_{0} e^{-j k_{0} r}}{\Pi r}\left\{\frac{\sin \left(\frac{k_{0} h}{2} \cdot \cos \phi\right)}{\frac{k_{0} h}{2} \cos \phi}\right\} \cos \left(\frac{k_{0} L e}{2} \sin \phi\right)
$$

E- plane $\left(\theta=90^{\circ}, 0^{\circ} \leq \phi \leq 90^{\circ}\right.$ and $\left.270^{\circ} \leq \phi \leq 360^{\circ}\right)$

$$
E_{e}{ }^{t}=+j \frac{k_{0} W V_{0} e^{-j b_{0}}}{\Pi r}\left\{\sin \theta \frac{\sin \left(\frac{k_{0} h}{2} \sin \theta\right)}{\frac{k_{0} h}{2} \sin \theta} \frac{\sin \left(\frac{k_{0} W}{2} \cos \theta\right)}{\frac{k_{0} W}{2} \cos \theta}\right\}
$$

H-plane $\left(\phi=0^{\circ}, 0^{\circ} \leq \theta \leq 180^{\circ}\right)$

F. Directivity of a single slot given as

$$
\begin{aligned}
D_{0}=\left(\frac{2 \pi W}{\lambda_{0}}\right)^{2} \frac{1}{I_{1}} & I_{1}=\int_{0}^{\Pi}\left[\frac{\sin \left[\frac{k_{0} W}{2} \cos \theta\right]}{\cos \theta}\right]^{\text {Where }} \sin ^{3} \theta d \theta
\end{aligned}
$$

\section{G. Reflection Coefficient $|\boldsymbol{\Gamma}|$}

Reflection coefficient given by

$$
\Gamma=\frac{V_{0}^{+}}{V_{0}^{-}}=\left(\frac{Z_{L}-Z_{0}}{Z_{L}+Z_{0}}\right)
$$

$\mathrm{V}_{0}^{+}$incident voltage

$\mathrm{V}_{0}$ voltage of reflection

Where $|\Gamma|$ is the reflection coefficient

\section{H. Voltage Standing Wave Ratio(VSWR)}

$$
V S W R=\frac{V_{\max }}{V_{\min }}=\frac{(1+|\Gamma|)}{(1-|\Gamma|)}
$$

VSWR ranges from 1 to $\infty$.

\section{Return Loss}

$$
R L=-20 \log |\Gamma|(d B)
$$

Where $|\Gamma|$ is the reflection coefficient

\section{RECTANGULAR MICROSTRIP PATCH ARRAY}

This paper presenting two different arrays .

A.The Arrayfactor of an $\mathrm{N}$-element linear array of isotropic sources is

$$
\begin{gathered}
A F=1+e^{i k d \cos \theta}+e^{i k 2 d \cos \theta}+\ldots \ldots . . e^{j k(N-1) d \cos \theta} \\
=\sum_{m=0}^{N-1} e^{i k m d \cos \theta}=\sum_{m=0}^{N-1} e^{j k m \frac{d}{N-1} \cos \theta}
\end{gathered}
$$

For the rectangular array antenna, the principal E-plane in $x-y$ is given by

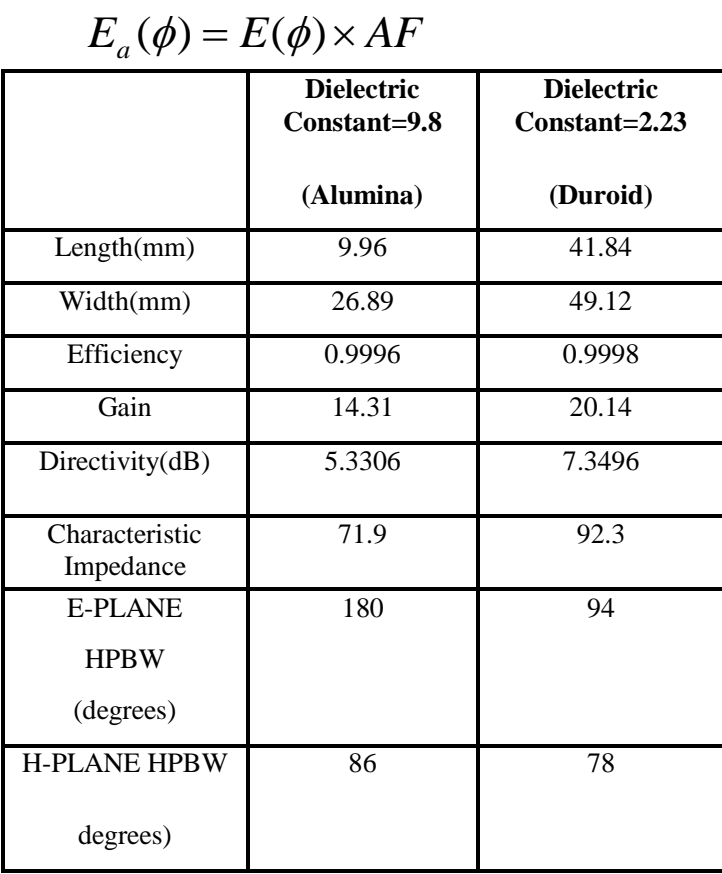

$$
=\frac{\sin \left(\frac{k_{0} h}{2} \cos \phi\right)}{\frac{k_{0} h}{2} \cos \phi} \sin \left(\frac{k_{0} L}{2} \cos \phi\right) \times \frac{\sin \left(\frac{N \psi}{2}\right)}{\sin \left(\frac{\psi}{2}\right)}
$$

\section{B. Dolph-Tschebyscheff Array.}

For a broadside array $(\beta=0), \mathrm{P}=2 \mathrm{M}($ even $)$

$$
\begin{gathered}
(A F)_{e}=2 \sum_{n-1}^{M} a_{n} \cos \left[\left(\frac{2 n-1}{2}\right) \frac{\pi d}{\lambda} \cos \theta\right] \\
u=\frac{\pi d}{\lambda} \cos \theta
\end{gathered}
$$

\section{RESULTS AND CONCLUSIONS:}

Rectangular micro strip patch antenna output parameters are designed using S-band frequency which is used for Wi-Fi applications. The sizing of the antenna are the width(W) ,Length (L) and the performance parameters of the antenna are shown in Table1, observed that with $\varepsilon_{\mathrm{r}}=2.23$, the size of 
the antenna $(\mathrm{L}=41.84 \mathrm{~mm}, \mathrm{~W}=49.12 \mathrm{~mm})$ increased. Directivity of the antenna is very high $=7.35 \mathrm{~dB}$. With the 9.8(Alumina), the sizeof the antenna $(\mathrm{L}=9.96 \mathrm{~mm}$, $\mathrm{W}=26.89 \mathrm{~mm}$ ) reduced, and the Directivity of the antenna is low $=5.33 \mathrm{~dB}$.

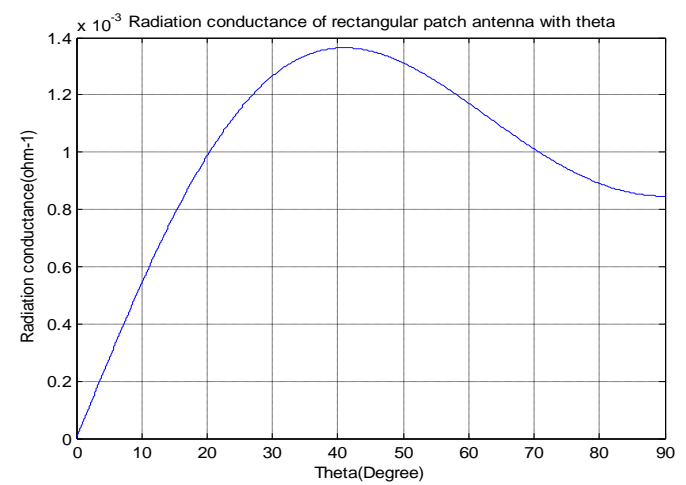

Fig. 4. Variation of Radiation in the rectangular microstrip patch with Theta.

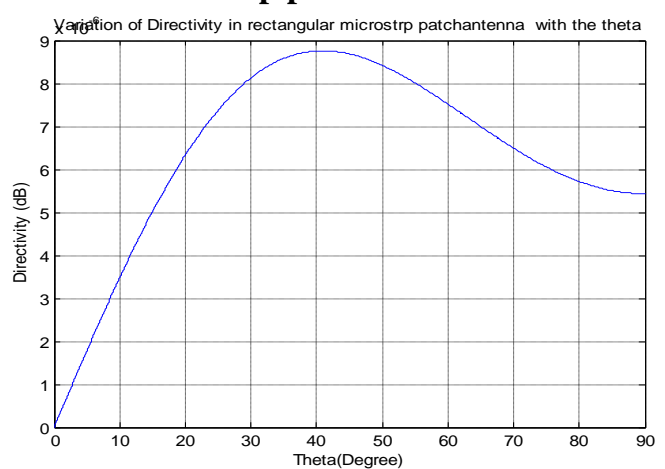

Fig. 5. Variation of Directivity in the rectangular microstrip patch with Theta.

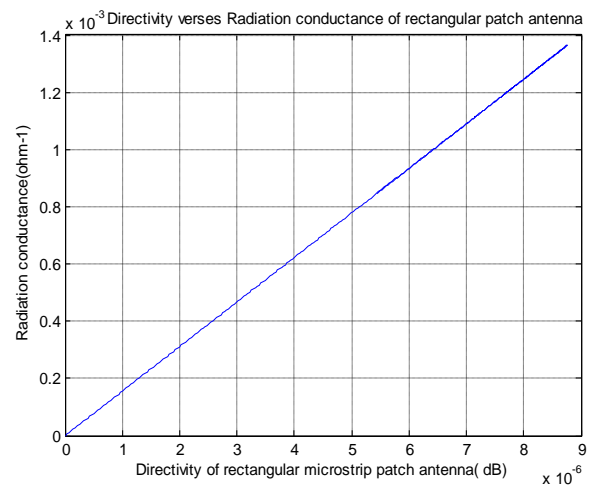

Fig. 6. Variation of Radiation Conductance with Directivity of rectangular patch

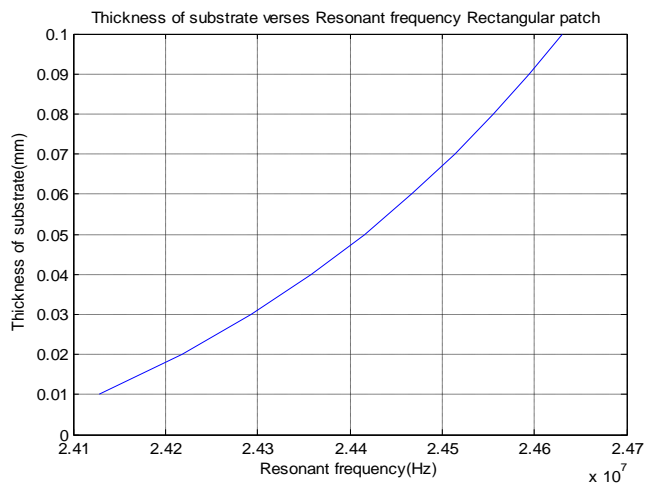

Fig. 7. Plot of variation of Thickness of substrate with Resonant frequency of rectangular microstrip patch.

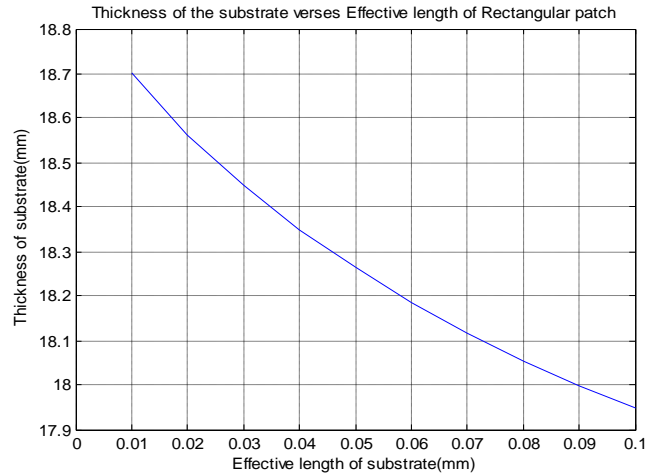

Fig. 8. Plot of variation of Thickness of substrate with Effective length.

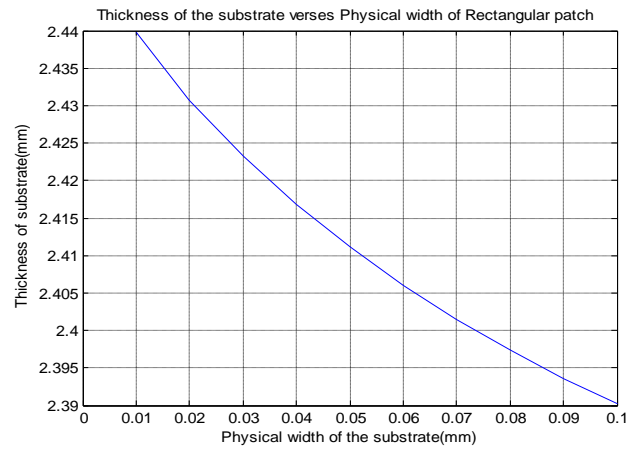

Fig. 9. Plot of variation of Thickness of substrate with Physical width of the substrate for rectangular microstrip patch.

Performance parameters of rectangular patch antenna are VSWR and Returnloss, are simulated.

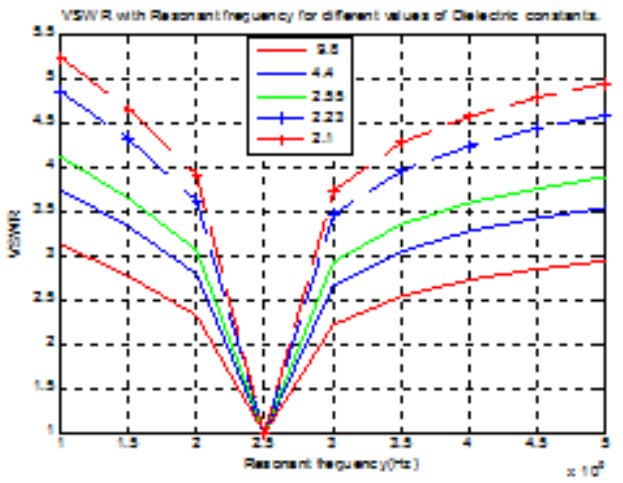

Fig. 10..Variation of VSWRof rectangular microstrip patch with respect to frequency.

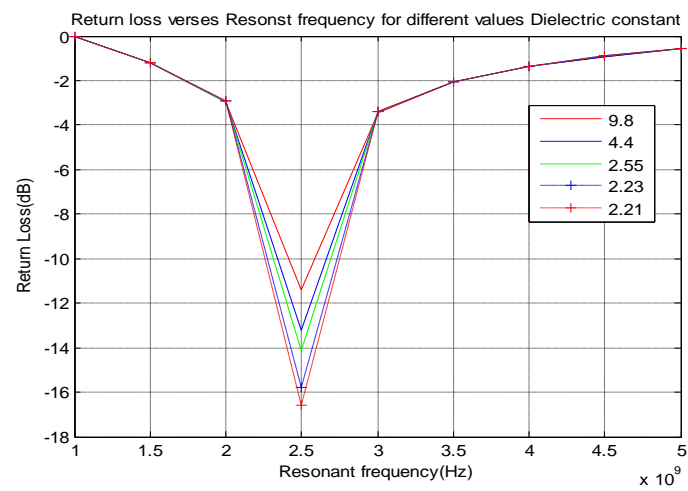

Fig .11. Variation of Return losses of rectangular microstrip patch at $2.5 \mathrm{GHz}$. 


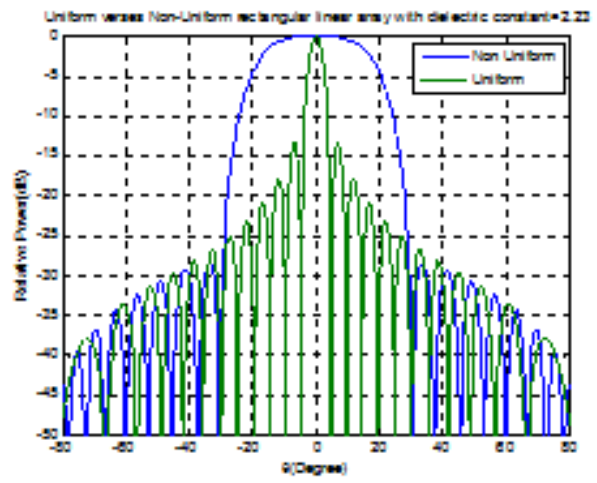

Fig. 12. 20 element rectangular microstrip linear array with Dieleectric constant $\left(\varepsilon_{\mathrm{r}}\right)=\mathbf{2 . 2 3}$.

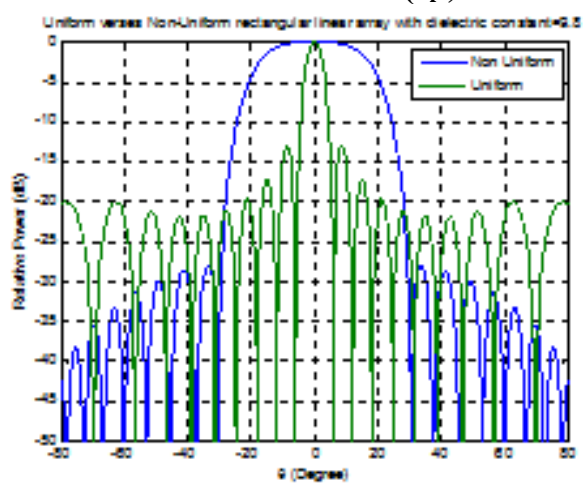

Fig. 13. 20 element rectangular microstrip linear array with $\varepsilon_{\mathrm{r}}=9.8$.

Table.2.Comparison between uniform \& non uniform rectangular microstrip array antenna Side Lobe Level (SLL) with different values of substrate dielectric constant $\left(\varepsilon_{\mathbf{r}}\right)$ values.

\begin{tabular}{|c|c|c|c|c|}
\hline $\begin{array}{l}\text { Dielectric } \\
\text { constant } \\
\left(\varepsilon_{\mathbf{r}}\right)\end{array}$ & $\begin{array}{c}\text { Uniform rectangular } \\
\text { linear array }\end{array}$ & \multicolumn{2}{|c|}{$\begin{array}{c}\text { Non uniform } \\
\text { rectangular } \\
\text { linear array }\end{array}$} \\
\hline & $\begin{array}{c}H P B W \\
(D e g .\end{array}$ & $\begin{array}{c}P S L L \\
(d B)\end{array}$ & $\begin{array}{c}H P B W \\
(\text { Deg. }\end{array}$ & $\begin{array}{c}P S L L \\
(d B)\end{array}$ \\
\hline 1 & 4.22 & -13.36 & 35.1 & -30 \\
\hline 2.23 & 4.42 & -13.28 & 35.8 & -28.48 \\
\hline 4.4 & 5.02 & -13.20 & 36.24 & -28.2 \\
\hline 9.8 & 5.30 & -13.07 & 36.42 & -28.06 \\
\hline
\end{tabular}

Table 2. shows the Comparison between uniform \& non uniform rectangular microstrip array antenna Peak Sidelobe Level (SLL) with different values of substrate dielectric $\operatorname{constant}\left(\varepsilon_{\mathrm{r}}\right)$.

From all the simulated results from the table 2, the rectangular micro strip antenna with non uniform array, with lower values of $\varepsilon_{\mathrm{r}}$ is preferred to get, reduced Peak Sidelobe Level, HPBW, FNBW, good voltage standing ratio and good Returnlosses, this designed rectangular array is good choice to used in Wi-Fi Modems.

\section{REFERENCES}

1. G. O. Young, "Synthetic structure of industrial plastics (Book style with paper title and editor)," in Plastics, 2nd ed. vol. 3, J. Peters, Ed. New York: McGraw-Hill, 1964, pp. 15-64.

2. W.-K. Chen, Linear Networks and Systems (Book style). Belmont, CA: Wadsworth, 1993, pp. 123-135.

3. H. Poor, An Introduction to Signal Detection and Estimation. New York: Springer-Verlag, 1985, ch. 4.

4. B. Smith, "An approach to graphs of linear forms (Unpublished work style)," unpublished.

5. E. H. Miller, "A note on reflector arrays (Periodical style-Accepted for publication)," IEEE Trans. Antennas Propagat., to be published.
6. J. Wang, "Fundamentals of erbium-doped fiber amplifiers arrays (Periodical style-Submitted for publication)," IEEE J. Quantum Electron., submitted for publication.

7. C. J. Kaufman, Rocky Mountain Research Lab., Boulder, CO, private communication, May 1995.

8. Y. Yorozu, M. Hirano, K. Oka, and Y. Tagawa, "Electron spectroscopy studies on magneto-optical media and plastic substrate interfaces(Translation Journals style)," IEEE Transl. J. Magn.Jpn., vol. 2, Aug. 1987, pp. 740-741 [Dig. $9^{\text {th }}$ Annu. Conf. Magnetics Japan, 1982, p. 301].

9. M. Young, The Techincal Writers Handbook. Mill Valley, CA: University Science, 1989.

10. (Basic Book/Monograph Online Sources) J. K. Author. (year, month, day). Title (edition) [Type of medium]. Volume(issue). Available: http://www.(URL)

11. J. Jones. (1991, May 10). Networks (2nd ed.) [Online]. Available: http://www.atm.com

12. (Journal Online Sources style) K. Author. (year, month). Title. Journal [Type of medium]. Volume(issue), paging if given. Available: http://www.(URL)

\section{AUTHORS PROFILE}

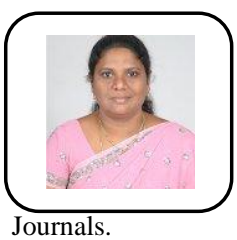

K.Karuna Kumari did her M.E in ECE in Andhra University. She has been working in the faculty of ECE in GITAM University for the last 14 years. She is pursuing his $\mathrm{Ph} . \mathrm{D}$. in the field of Optimization of microstrip antenna arrays in the Department of ECE, AU. She has published 14 papers in International

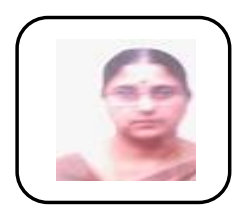

Dr.P.V.Sridevi honored with Ph.D from AUCE, Andhra University, Visakhapatnam, A.P, India in 1997. She published many research papers in different international/national journals and also conferences. Presently she is a professor in the Dept. of ECE, AUCE, Andhra University, Visakhapatnam, India. She guided $10 \mathrm{Ph} . \mathrm{D}$ theses in the fields of Antennas, Electro Magnetics, EMI/EMC and Microwave, Radar Communications, VLSI. She is having 31 years of experience in teaching and 20 years in research. 